\title{
Estrategias de lectura y su relación con la comprensión lectora en los estudiantes del III ciclo académico - Facultad de Ciencias Sociales, Educación y de la Comunicación - Universidad Nacional Santiago Antúnez de Mayolo - Huaraz, 2017
}

\section{Reading strategies and their relationship with the reading comprehension in the students of the III academic cycle - Faculty of Social Sciences, Education and Communication - National University Santiago Antunez of Mayolo - Huaraz, 2017}

\author{
Fernando Trujillo Castillo ${ }^{1}$, Abelardo Rodolfo Campana Concha
}

\begin{abstract}
RESUMEN
Objetivo: Determinar la relación de las Estrategias de Lectura y su relación con la Comprensión Lectora de los estudiantes del III semestre académico de la Facultad de Ciencias Sociales, Educación y de la Comunicación - Universidad Nacional "Santiago Antúnez de Mayolo" - Huaraz 2017. Material y Métodos: Se planteó el enfoque cuantitativo de diseño correlacional, asimismo se recolectó información mediante un cuestionario y un test de conocimientos que fue aplicado a la muestra integrado por 100 estudiantes. De otro lado, se garantizó su confiabilidad mediante la prueba de Alfa de Cronbach, además de aplicar el paquete computacional SPSS versión 21, con el empleo de la fórmula de Rho de Spearman con un margen de error al 5\%. Resultados: La hipótesis general representado por las variables de estudio encontró una correlación buena de Rho = 790 , ya que la mayoría consideró que las estrategias de lectura son aplicadas a veces, lo cual afectó a la comprensión lectora que alcanzó un nivel académico de regular. Ese mismo procedimiento se aplicó en las tres hipótesis específicas cuyos resultados determinaron una correlación de Rho = $.610, \mathrm{Rho}=.567$ y Rho $=, 599$. Conclusión: Las estrategias de planificación, monitoreo y evaluación no son aplicadas con frecuencia lo cual impide una adecuada comprensión en los textos académicos.
\end{abstract}

Palabras clave: Estrategias de lectura, comprensión lectora, planificación, monitoreo, evaluación.

\section{ABSTRACT}

Objective: To determine the relationship between the Reading Strategies and their relationship with the Reading Comprehension of the students of the III academic semester of the Faculty of Social Sciences, Education and Communication - National University "Santiago Antúnez de Mayolo" Huaraz 2017. Material and Methods: The quantitative approach of correlational design was raised, information was also collected through a questionnaire and a knowledge test that was applied to the sample made up of 100 students. On the other hand, its reliability was guaranteed by the Cronbach's Alpha test, in addition to applying the SPSS version 21 computational package, with the use of Spearman's Rho formula with a margin of error of $5 \%$. Results: The general hypothesis represented by the study variables found a good correlation of Rho $=.790$, since most considered that reading strategies are sometimes applied, which affected the reading comprehension that reached a regular academic level. That same procedure was applied in the three specific hypotheses whose results determined a correlation of Rho $=.610$, Rho $=.567$ and Rho $=, 599$. Conclusion: The planning, monitoring and evaluation strategies are not frequently applied, which prevents an adequate understanding in the academic texts.

Keywords: Reading strategies, reading comprehension, planning, monitoring, evaluation.

\section{INTRODUCCIÓN}

El presente estudio se realizó motivado por el bajo nivel de comprensión lectora mostrado en los resultados de los últimos exámenes para el nombramiento, contrato de docentes y educandos de niveles básicos en la región Ancash y a nivel nacional (ECE y PISA). Las pruebas de comprensión lectora se programan solo para los exámenes de admisión; después de ella no se toman en cuenta los criterios formulados en dichas pruebas tales como: desarrollar las competencias y capacidades para reconstruir los pensamientos que transmite el autor sobre los hechos reales o ficticios; motivar una actitud analítica que permita conocer mejor la realidad, enfrentar las adversidades actuales y prever las futuras; mejorar y ampliar nuestra visión del mundo, acceder a una formación segura, autorizada y permanente, incrementar nuestro vocabulario y dominar el empleo adecuado de los términos. Las circunstancias actuales de la educación en nuestro país, expresan un conjunto de demandas urgentes, tanto políticas, económicas, sociales y técnicas. Además, viendo los resultados alarmantes de las pruebas PISA, donde nuestro país se ubica en los últimos puestos a nivel internacional y en Latinoamérica, en cuanto se refiere a la comprensión lectora, matemáticas y ciencias, desarrollados a partir del año 2000 a 2015. Así como también en la ECE (Evaluación Censal de Estudiantes) realizados en estos últimos años, conocido que el rendimiento académico en la actualidad está en tela de juicio, no encontrándose las verdaderas causas.

Comparando los resultados obtenidos por el Perú en Comprensión Lectora entre las pruebas PISA2000, PISA 2009 y 2015 se observa que hubo una leve mejora, pero que todavía le coloca en un lugar bajo del ranking internacional. Algo que debe destacarse es que en Comprensión Lectora se pasó de

'Estudiante de Maestría de la Universidad Nacional Mayor de San Marcos ${ }^{2}$ Docente de Educación de la Universidad Nacional Mayor de San Marcos
327 a 370 puntos, constituyéndose en el país que más mejoró (con respecto a sí mismo) entre las 61 naciones participantes. El puntaje promedio de los países americanos en PISA 2009 fue de 408. (MINEDU, 2017).

Incluso el INEI (2012) también brinda algunos detalles de la realidad educativa escolar, en donde detalla que un $30,9 \%$ de ellos están en un nivel suficiente, $49,3 \%$ en proceso y $19,3 \%$ en inicio. en consecuencia, podrían presentar dificultades académicas en futuro universitario, ya que las bases académicas en ellos deben ser fuertes para asumir idóneamente los textos universitarios.

Nos parece bien que haya metas educativas nacionales; sin embargo, es conveniente que el MINEDU fije, también metas a lograr en las pruebas PISAal 2021

Sería un avance que en la última prueba en Comprensión Lectora se haya obtenido 390 puntos, la proyección al 2021 sería obtener alrededor de 450 puntos, lo que le podría permitir a nuestro país estar en el grupo promedio de todas las naciones que participen en dicha evaluación internacional.

Naturalmente, el Estado debe priorizar y desarrollar políticas y estrategias para transitar todos juntos con optimismo y esperanza el complejo camino que nos posibilite lograr o acercarnos a las metas educativas propuestas en Comprensión Lectora.

Debido a estos resultados preocupantes, para el presente estudio, se toma en cuenta los temas de mayor trascendencia relacionados a las estrategias de lectura y el nivel de comprensión lectora. De esta manera se propone conocer las relaciones entre las mencionadas variables presentados por los estudiantes en el medio.

Recibido:27/06/19 Aprobado:09/08/19 
Asimismo, es importante la realización de estudios, referente a las estrategias de lectura y la comprensión lectora, debido a las dificultades en la comprensión de textos escritos y que desde los niveles de educación inicial se den mucha importancia a estos temas para mejorar paulatinamente la comprensión y el rendimiento académico de los educandos.

Cabe mencionar que en los últimos años se ha perdido demasiado el hábito (gusto - placer) por la lectura, siendo ello uno de los factores para el bajo nivel de comprensión lectora, asimismo la falta de conocimiento del uso adecuado de las estrategias de lectura.

En la actualidad, elevar el nivel de comprensión lectora de los educandos de niveles superiores y básicos debe ser un reto para las universidades y el Estado peruano, donde los programas curriculares deben de estar acordes a las necesidades e intereses de los educandos y los retos de los avances científicos y tecnológicos de estos tiempos. Por lo tanto, las universidades y centros de formación docente, deben desarrollar las competencias comunicativas textuales para fortalecer las capacidades y habilidades en la comprensión lectora de nuevos egresados.

Por tanto el objetivo de nuestra investigación fué el demostrar la relación existente entre las Estrategias de Lectura y la Comprensión Lectora en los estudiantes del III semestre académico de la Facultad de Ciencias Sociales, Educación y de la Comunicación - Universidad Nacional "Santiago Antúnez de Mayolo" - Huaraz 2017.

\section{MÉTODOS Y MATERIALES}

El tipo de investigación es correlacional con un enfoque cuantitativa de nivel explicativo, donde se analiza la relación de dos variables: Estrategias de Lectura y la Comprensión Lectora

\section{Población}

La población está conformada por 100 estudiantes del III Semestre Académico - Facultad de Ciencias Sociales, Educación y de la Comunicación - Universidad Nacional Santiago Antúnez De Mayolo - Huaraz, 2017.

Se aplicaron los instrumentos, como el cuestionario para la variable X: Estrategias de Lectura, mientras que la variable Y: Comprensión Lectora se aplicó el Test de conocimientos.

Para el tratamiento estadístico, se empleó el paquete computacional SPSS versión 21 , se estableció la contratación de las hipótesis.

\section{RESULTADOS}

\section{Prueba de hipótesis general}

HGA. Existe una relación significativa entre las Estrategias de Lectura y la Comprensión Lectora en los estudiantes del III semestre académico de la Facultad de Ciencias Sociales, Educación y de la Comunicación - Universidad Nacional "Santiago Antúnez de Mayolo" - Huaraz 2017.

HGO. No existe una relación significativa entre las Estrategias de Lectura y la Comprensión Lectora en los estudiantes del III semestre académico de la Facultad de Ciencias Sociales, Educación y de la Comunicación - Universidad Nacional "Santiago Antúnez de Mayolo" - Huaraz 2017.

\section{Tabla 1}

Correlación de la hipótesis general

Comprensión lectora

\begin{tabular}{llcr} 
& & \multicolumn{2}{c}{ Comprensión lectora } \\
\hline $\begin{array}{l}\text { Rho de } \\
\text { Spearman }\end{array}$ & $\begin{array}{c}\text { Estrategias } \\
\text { de lectura }\end{array}$ & $\begin{array}{c}\text { Coeficiente de } \\
\text { correlación }\end{array}$ & 0,790 \\
& & Sig. (bilateral) & 0,000 \\
& $\mathrm{~N}$ & 100 \\
\hline
\end{tabular}

Interpretación: Al aplicar la fórmula de Rho de Spearman, margen de error al $5 \%$, se halló un coeficiente de correlación buena de Rho $=0,790$ y el $p=0,000$. Por lo cual se rechaza la hipótesis nula y se acepta la hipótesis alterna, cuyos resultados fueron determinados por la respuesta de la muestra, en donde la mayoría considera que las Estrategias de lectura, identificado mayoritariamente como a veces aplicadas en sus actividades académicas, interfiere en la compresión lectora.

\section{Contrastación de la primera hipótesis específica}

H1. La Planificación se relaciona significativamente con la Comprensión Lectora en los estudiantes del III semestre académico de la Facultad de Ciencias Sociales, Educación y de la Comunicación - Universidad Nacional "Santiago Antúnez de Mayolo"-Huaraz, 2017.

Ho. La Planificación no se relaciona significativamente con la Comprensión Lectora en los estudiantes del III semestre académico de la Facultad de Ciencias Sociales, Educación y de la Comunicación - Universidad Nacional "Santiago Antúnez de Mayolo"-Huaraz, 2017.

Tabla 2

Correlación de la primera hipótesis específica

\begin{tabular}{lcr}
\hline & \multicolumn{2}{c}{ Comprensión lectora } \\
\hline & \multicolumn{2}{c}{$\begin{array}{c}\text { Coeficiente de } \\
\text { correlación }\end{array}$} \\
$\begin{array}{l}\text { Rho de } \\
\text { Spearman }\end{array}$ & Planificación & 0,610 \\
& Sig. (bilateral) & 0,000 \\
& $\mathrm{~N}$ & 100 \\
\hline
\end{tabular}

Interpretación: Al aplicar la fórmula de Rho de Spearman, margen de error al $5 \%$, se halló un coeficiente de correlación buena de $R$ ho $=0,610$ y el $p=0,000$. Por lo cual se rechaza la hipótesis nula y se acepta la hipótesis alterna, cuyos resultados fueron determinados por la respuesta de la muestra, en donde la mayoría considera que la Planificación, identificado mayoritariamente como a veces aplicadas en sus actividades académicas, interfiere en la compresión lectora.

\section{Contrastación de la segunda hipótesis específica}

$\mathrm{H} 2$. El monitoreo se relaciona significativamente con la Comprensión Lectora en los estudiantes del III semestre académico de la Facultad de Ciencias Sociales, Educación y de la Comunicación - Universidad Nacional "Santiago Antúnez de Mayolo"-Huaraz, 2017.

Ho. El monitoreo no se relaciona significativamente con la Comprensión Lectora en los estudiantes del III semestre académico de la Facultad de Ciencias Sociales, Educación y de la Comunicación - Universidad Nacional "Santiago Antúnez de Mayolo"-Huaraz, 2017.

Tabla 3

Correlación de la segunda hipótesis especifica

\begin{tabular}{|c|c|c|c|}
\hline & & \multicolumn{2}{|c|}{ Comprensión lectora } \\
\hline \multirow{3}{*}{$\begin{array}{l}\text { Rho de } \\
\text { Spearman }\end{array}$} & \multirow{3}{*}{ Planificación } & $\begin{array}{l}\text { Coeficiente de } \\
\text { correlación }\end{array}$ & 0,567 \\
\hline & & Sig. (bilateral) & 0,000 \\
\hline & & $\mathrm{N}$ & 100 \\
\hline
\end{tabular}

Interpretación: Al aplicar la fórmula de Rho de Spearman, margen de error al $5 \%$, se halló un coeficiente de correlación moderada de $R h o=0,567$ y el $p=0,000$. Por lo cual se rechaza la hipótesis nula y se acepta la hipótesis alterna, cuyos resultados fueron determinados por la respuesta de la muestra, en donde la mayoría considera que el Monitoreo, identificado mayoritariamente como a veces aplicadas en sus actividades académicas, interfiere en la compresión lectora. 


\section{Contrastación de la tercera hipótesis específica}

H3. La evaluación se relaciona significativamente con la Comprensión Lectora en los estudiantes del III semestre académico de la Facultad de Ciencias Sociales, Educación y de la Comunicación - Universidad Nacional "Santiago Antúnez de Mayolo"-Huaraz, 2017

Ho. La evaluación no se relaciona significativamente con la Comprensión Lectora en los estudiantes del III semestre académico de la Facultad de Ciencias Sociales, Educación y de la Comunicación - Universidad Nacional "Santiago Antúnez de Mayolo"-Huaraz, 2017

Tabla 4

Correlación de la tercera hipótesis especifica

\begin{tabular}{|c|c|c|c|}
\hline & & \multicolumn{2}{|c|}{ Comprensión lectora } \\
\hline \multirow{3}{*}{$\begin{array}{l}\text { Rho de } \\
\text { Spearman }\end{array}$} & \multirow{3}{*}{ Planificación } & $\begin{array}{l}\text { Coeficiente de } \\
\text { correlación }\end{array}$ & 0,599 \\
\hline & & Sig. (bilateral) & 0,000 \\
\hline & & $\mathrm{N}$ & 100 \\
\hline
\end{tabular}

Interpretación: Al aplicar la fórmula de Rho de Spearman, margen de error al $5 \%$, se halló un coeficiente de correlación moderada de $R h o=0,599$ y el $p=0,000$. Por lo cual se rechaza la hipótesis nula y se acepta la hipótesis alterna, cuyos resultados fueron determinados por la respuesta de la muestra, en donde la mayoría considera que la Evaluación, identificado mayoritariamente como a veces aplicadas en sus actividades académicas, interfiere en la compresión lectora.

\section{DISCUSIÓN}

En la hipótesis general del presente estudio, se determinó estadísticamente un coeficiente de correlación buena de Rho = 0,790 , entre las variables Estrategias de Lectura y la Comprensión Lectora en la población de estudio. Se halló respuestas divididas de parte de la muestra, representadas en la variable Estrategias de Lectura cuyas respuestas determinaron que el $56.0 \%$ lo describió como a veces, seguido de un $24,0 \%$ que lo identifica como casi siempre. Asimismo, para la variable Comprensión Lectora cuyas respuestas también se encuentran divididas, puesto que el $48 \%$ alcanzó un nivel regular, mientras que un $26,0 \%$ obtuvo un nivel bueno. Con ello se concluye que las estrategias de lectura aplicado por los estudiantes son insuficientes para que la comprensión lectora alcance los objetivos académicos.

En cuanto a la primera hipótesis específica se estableció en las estadísticas un coeficiente de correlación buena de Rho = ,610 entre la Planificación y la variable Comprensión Lectora de los estudiantes de la población en estudio. Se pudo encontrar diferencias en la muestra, representadas en la dimensión Planificación cuyas respuestas determinaron que el 53\% lo identifica como a veces, seguido de un $31 \%$ que lo refiere como casi siempre. Asimismo, para la variable Comprensión Lectora cuyas respuestas también se encuentran divididas, puesto que el $48 \%$ alcanzó un nivel regular, mientras que un $26,0 \%$ obtuvo un nivel bueno. Con lo cual se confirma que la planificación aplicada por los estudiantes resulta insuficiente para que la comprensión lectora alcance los objetivos académicos.

En la segunda hipótesis específica, las estadísticas encontraron un coeficiente de correlación moderada de Rho = .567 , entre la dimensión Monitoreo y la Comprensión Lectora de los estudiantes. Encontrándose diferencias en las respuestas de la muestra en cuanto a la dimensión Monitoreo cuyas respuestas determinaron que el $56,0 \%$ lo identifica como a veces, seguido de un $26,0 \%$ que lo refiere como casi siempre. Del mismo modo, para la variable Comprensión Lectora cuyas respuestas también se encuentran divididas, puesto que el 48
\% alcanzó un nivel regular, mientras que un $26,0 \%$ obtuvo un nivel bueno. Por ello, se confirma que monitoreo aplicado por los estudiantes son insuficientes para que la comprensión lectora alcance los objetivos académicos.

En la tercera hipótesis específica, las estadísticas hallaron un coeficiente de correlación moderada de Rho $=.599$, entre la dimensión Evaluación y la Comprensión Lectora de los estudiantes. Encontrándose diferencias en las respuestas de la muestra en cuanto a la dimensión Evaluación cuyas respuestas precisaron que el $59,0 \%$ lo identifica como a veces, seguido de un $23,0 \%$ que lo refiere como casi siempre. Asimismo, para la variable Comprensión Lectora cuyas respuestas también se encuentran divididas, puesto que el $48 \%$ alcanzó un nivel regular, mientras que un $26,0 \%$ obtuvo un nivel bueno. Confirmándose que la evaluación resulta insuficiente para obtener mejores resultados académicos en cuanto a la comprensión lectora.

Los resultados encontrados son similares a los de Neira (2015) que demuestran que la comprensión lectora se ve afectada por las características de los estudiantes solo cuando se leen textos de carácter general. Sin embargo, cuando se leen textos especializados, propios de su carrera, no se observan diferencias significativas. Asimismo, detalla que los lectores usan distintas estrategias y con distinta frecuencia para enfrentar los textos de carácter general y los textos especializados. Esto se observó en las diferencias entre las estrategias empleadas en la lectura de un cuento y la de un artículo especializado, las que dejan en evidencia que el procesamiento del primero se realiza de abajo-arriba (bottomup) y el procesamiento del segundo ocurre de arriba-abajo (topdown). En otras palabras, notamos cierta motivación lectora en los textos académicos implementados en su carrera, pero textos ajenos a este se les presenta mayor dificultad, lo cual da cuenta que aún se presentan deficiencias para aplicar estrategias y tener una comprensión adecuada. Hemos podido comprobar, que las deficiencias lectoras de nuestra población en estudio, manifestaron que la frecuencia en la que aplican estrategias para leer es de a veces, incidiendo en la comprensión lectora de los diversos textos académicos. Por lo cual, vemos que la lectura, aún no es vista como un hábito, sino como una necesidad de terminar una carrera universitaria que facilite aprobar los cursos que se les imparte a los estudiantes, quienes ignoran la importancia de conocer nuevos textos.

En las investigaciones de Neira (2015) se ha demostrado que los inconvenientes para resultados favorables, es la ausencia de implementación de una Metodología interactiva que permite englobar los aspectos Bottom-up y Top-down, el cual permite la aplicación de estos aspectos, es decir, que en el proceso de lectura se aplique la descodificación de las palabras y las frases, así como también de la recurrencia de algunas estrategias necesarias para procesar activamente el texto, mediante los conocimientos previos que posee el lector. Sin embargo, también nos dan luces de la necesidad de que el docente proponga nuevas metodologías de lectura, mientras imparte sus clases.

Con respecto a la comprensión lectora en los universitarios, nos encontramos con los estudios desarrollados por Palacios (2015) quien es sus conclusiones destaca que los estudiantes carecen de hábitos de lectura, ya que presentaron deficiencias en establecer de manera firme la relación entre el título y el contenido del texto, asimismo establecieron una inferencia final e identificar la intensión del autor, técnicas de leer y escribir. Del mismo modo, señalan que el desarrollo de estrategias de lectura en los estudiantes universitarios fomenta en ellos, su autonomía en el proceso de comprensión, así como también, le exige una mayor demanda cognitiva al extraer el sentido del texto que lee. 
Sin embargo, esta investigación nos ayuda a reiterar que las estrategias de lectura no son frecuentes en los estudiantes universitarios, a lo que Palacios (2015) refiere que les cuesta demasiado aplicarlos porque les demanda mucho esfuerzo cognitivo, sin embargo, también es necesario entender que esa dificultad puede presentarse desde antes, si no tiene una base estrategia de lectura reforzada desde la etapa escolar existe mayor posibilidad de la presencia de dificultades en un nivel superior.

\section{CONCLUSIONES}

La poca frecuencia en la cual los estudiantes recurren a las estrategias de lectura disminuye las posibilidades de comprender textos académicos que beneficien a su formación académica.

Si los estudiantes no incrementan la frecuencia en la que planifican el propósito de la lectura y sus objetivos en base a las necesidades de cada curso, disminuye las posibilidades de comprender adecuadamente los textos académicos que beneficien a su formación académica.

La poca frecuencia en la elaboración de sus propios conceptos mediante la inferencia o deducción por parte de los estudiantes, disminuye las posibilidades de comprender adecuadamente los textos académicos que beneficien a su formación académica.

\section{RECOMENDACIONES}

A las autoridades universitarias, incentivar programas $y / 0$ actividades académicas que fomenten la lectura en los estudiantes, de esta manera ellos puedan reforzar las estrategias de lectura que más se adapte a sus necesidades e intereses para lograr una adecuada compresión de textos.

A los docentes se les sugiere motivar constantemente a los estudiantes a tener mayor acercamiento con la lectura, no solo por textos específicamente académicos, sino de su interés personal, de esta manera tendrán mayor facilidad de identificar el propósito de la lectura y sus objetivos de este, de esta manera ayuda al estudiante a reforzar esta estrategia para que pueda ser aplicada en futuras ocasiones.
Se recomienda a los estudiantes concientizar sobre la importancia de reforzar las estrategias de monitoreo, ya que es común no entender un determinado texto en el primer intento ya sea por su complejidad o su extensión, por eso, es importante encontrar la forma y la manera, que más se nos acomode, para resaltar las ideas importantes, de esta manera los ayudará a releer el texto de la forma más rápida y las ideas necesarias para extrapolar ideas. Es por eso, que los estudiantes deben ser responsables que la comprensión de lectura parte de su reforzamiento constante.

Los docentes deben desarrollar actividades más participativas en clases para reforzar las estrategias de evaluación, en donde pueda exigirles a los estudiantes un análisis con respecto al texto y sus propios conocimientos. Asimismo, se tiene que dejar de lado practicas memorísticas dentro del aula, por eso el debate resulta una importante alternativa para generar la participación y el intercambio de ideas.

\section{REFERENCIAS BIBLIOGRÁFICAS}

MINEDU (2017). El Perú en PISA 2015 Informe nacional de resultados, Lima, Perú. Recuperado en: h t t p://um c.minedu.gob.pe/wp content/uploads/2017/04/Libro PISA.pdf

INEI (2012). Perú: Indicadores de Educación por Departamentos, 2001-2012. Recuperado en: https://www.inei.gob.pe/media/MenuRecursivo/public aciones_digitales/Est/Lib1150/cap08.pdf 